\title{
Evolution of Experience of Care of Patients with and without Chronic Diseases following a Québec Primary Healthcare Reform
}

\author{
Raynald Pineault, ${ }^{1,2,3,4}$ Roxane Borgès Da Silva, ${ }^{1,4,5}$ Sylvie Provost, ${ }^{1,3,5}$ \\ Mylaine Breton, ${ }^{6,7}$ Pierre Tousignant, ${ }^{1,8}$ Michel Fournier, ${ }^{1}$ \\ Alexandre Prud'homme, ${ }^{1}$ and Jean-Frédéric Levesque ${ }^{9,10}$ \\ ${ }^{1}$ Direction de Santé Publique de l'Agence de la Santé et des Services Sociaux de Montréal, 1301 Rue Sherbrooke Est, \\ Montréal, QC, Canada H2L 1M3 \\ ${ }^{2}$ Institut National de Santé Publique du Québec, 190 Boulevard Crémazie Est, Montréal, QC, Canada H2P 1E2 \\ ${ }^{3}$ Centre de Recherche du Centre Hospitalier de l'Université de Montréal, 900 Rue Saint-Denis, \\ Montréal, QC, Canada H2X 0A9 \\ ${ }^{4}$ Institut de Recherche en Santé Publique de l'Université de Montréal, 7101 Avenue du Parc, \\ Montréal, QC, Canada H3N $1 X 9$ \\ ${ }^{5}$ Faculté des Sciences Infirmières de l'Université de Montréal, 2375 Chemin de la Côte-Sainte-Catherine, \\ Montréal, QC, Canada H3T 1A8 \\ ${ }^{6}$ Centre de Recherche de l'Hôpital Charles LeMoyne, 150 Place Charles-LeMoyne, Bureau 200, Longueuil, \\ QC, Canada J5C $2 B 6$ \\ ${ }^{7}$ Département des Sciences de la Santé Communautaire, Université de Sherbrooke, 3001 12e Avenue Nord, \\ Sherbrooke, QC, Canada J1H $5 \mathrm{H} 3$ \\ ${ }^{8}$ Department of Epidemiology, Biostatistics and Occupational Health, McGill University, 1020 Avenue des Pins Ouest, \\ Montréal, QC, Canada H3A 1A2 \\ ${ }^{9}$ Centre for Primary Health Care and Equity, University of New South Wales, Level 3, AGSM Building, \\ Sydney, NSW 2033, Australia \\ ${ }^{10}$ Bureau of Health Information, 67 Albert Avenue, Chatswood, NSW 2067, Australia
}

Correspondence should be addressed to Raynald Pineault; rpineaul@santepub-mtl.qc.ca

Received 2 December 2015; Revised 16 February 2016; Accepted 22 February 2016

Academic Editor: Jens Klotsche

Copyright (C) 2016 Raynald Pineault et al. This is an open access article distributed under the Creative Commons Attribution License, which permits unrestricted use, distribution, and reproduction in any medium, provided the original work is properly cited.

Objectives. To assess the extent to which new primary healthcare (PHC) models implemented in two regions of Quebec have improved patient experience of care, unmet needs, and use of services for individuals with and without chronic diseases, compared with other forms of PHC practices. Methods. In 2005 and 2010, we carried out population and organization surveys. We divided PHC organizations into new model practices and other practices and followed the evolution over time of patient experience of care. Results. Patients with chronic diseases had better accessibility but worse continuity of care in the new model practices than in the other practices at both time periods. Through the reform, accessibility decreased evenly in both groups, but continuity and perceived outcomes improved more in the other practices. Use of primary care services decreased more in the new model practices. Among patients without chronic disease, accessibility decreased much less in the new models and responsiveness increased more. There was no significant change in ER attendance and hospitalization. Conclusion. The evolution of patient experience of care has been more favorable for patients without chronic diseases. These findings raise concerns about equity since the aim of the PHC reform was targeting in priority individuals with the greatest needs. 


\section{Introduction}

Increase in the number of the elderly and in the prevalence of chronic diseases presses healthcare systems to offer individuals with chronic diseases more comprehensive care through primary healthcare (PHC) [1-3]. To meet these needs, different models of chronic disease management have been proposed. The most widely known is the "Chronic Care Model" that advocates an integrated approach to care at all levels of the healthcare system for comprehensive and multidisciplinary management of chronic diseases [4]. It also advocates for the establishment of organizational mechanisms to support provision of services and interorganizational linkage to achieve better coordination and integration of services. Related to the "Chronic Care Model," other models have been proposed for the delivery of primary healthcare, including the "Patient-Centered Care Medical Home" [5-9]. All proposed models focus on common elements, including sharing of responsibilities between healthcare professionals in a multidisciplinary teamwork perspective, active role of individuals in monitoring of their diseases, importance of communication facilitated by a clinical information system, and integration of services in an organizational framework that supports clinical practices and fosters linkages with other components of healthcare systems $[3,10]$.

Based on these models, two new forms of PHC practices were created in the early 2000s in Quebec: Family Medicine Groups (FMGs) aimed at improving continuity of care and Network Clinics (NCs) intended to provide greater accessibility of services. A FMG is composed of 6 to 10 physicians with no geographical catchment area for patients they can register (between 10,000 and 15,000 patients by FMG). The FMG can count on a grant from the Ministry of Health and Social Services to support its operations, in exchange for a contractual engagement to register a predetermined number of patients and to provide a minimum of specified services. It also provides greater accessibility through extended hours and participation in a regional on-call system. As of November 2010, there were 217 accredited FMGs, including 25 in Montréal and 35 in Montérégie, the two regions that participated in this study. The NC is a complementary PHC practice model implemented in both regions, but mainly in Montréal at the initiative of the Regional Health and Social Services Agency. It specifically aimed to improve accessibility, through providing walk-in services and ensuring access to radiology and laboratory tests as well as medical specialist services. It receives financial support from the Regional Agency. In August 2010, there were 33 NCs in Montréal and 4 in Montérégie. The distinction between FMG and NC is often blurred, especially as some PHC practices have a dual FMG-NC status, thus benefiting from two sources of funding. The complementarity between FMGs and NCs was to allow the provision of more complete and better integrated care, particularly for individuals with chronic diseases [11-13]. In November 2010, 19 PHC practices had a FMG-NC status in Montréal and none in Montérégie.

Several studies have explored the association between structural features of PHC practices and experience of care
[14-18]. Studies have also assessed the impact of implementing specific components of the Chronic Care Model on various care processes and outcomes [19-21]. Few studies have looked at PHC practice organizational attributes to assess their potential for managing chronic diseases $[3,4]$. An Ontario study found that chronic disease management was superior in Community Health Centers compared to other types of practices mainly due to the presence of nurse practitioners and interdisciplinary teams [22]. In the context of a large study conducted in 2005 in two Quebec regions, we examined the overall patient experience of care in PHC practices and found a better continuity of care among practices that were more integrated and coordinated, but the model that focused on walk-in visits provided more accessible care $[13,23]$. This study reported on the situation prevailing in 2005 and did not address the impact of the new PHC practices as it was too early to assess the change. A related cohort study found that FMGs represented an integrated model of PHC delivery associated with higher levels of achievement in chronic care [24].

In close collaboration with the Regional Health and Social Services Agencies of the two most heavily populated Quebec regions, Montréal and Montérégie, we conducted a second study in 2010 to assess the evolution of the PHC reform and its impacts on patients' experience of care, unmet needs, and use of services [25].

The main objective of this paper is to assess the extent to which new PHC practice models implemented in the two regions (FMG-NC, FMG, and NC) have improved patient experience of care, unmet needs, and use of services for individuals with and without chronic diseases, compared with other forms of PHC practices that did not undergo such a change.

\section{Methods}

2.1. Design. The study design corresponds to a before-after natural experiment in which FMGs, NCs, and FMG-NCs constituted the experimental group and the other practices formed the control group (Figure 1). The study consisted of population and organization surveys conducted in 2005 and 2010 in Montréal and Montérégie [25]. At that time, the two regions were divided into 23 local territories (12 in Montréal and 11 in Montérégie) under the governance of Health and Social Services Centres. The population surveys were carried out on independent samples of the 2005 and 2010 populations. They included 9,206 adults aged 18 or more in 2005 with a response rate of $64 \%$ and 9,180 in 2010 with a response rate of $56 \%$. For the purpose of this study, we excluded respondents who did not have a regular source of care since only those who had a regular source of care reported on their experience of care. We also excluded those whose usual source of care was not identified at both time periods. These exclusions left 6,198 respondents in 2005 and 6,753 in 2010. Short telephone surveys of all PHC organizations were also carried out: 659 organizations in 2005 and 606 organizations in 2010. Basic information such as type of practice (solo, group, FMG, and NC), number of practicing family physicians, having a nurse in the clinic, and offering 


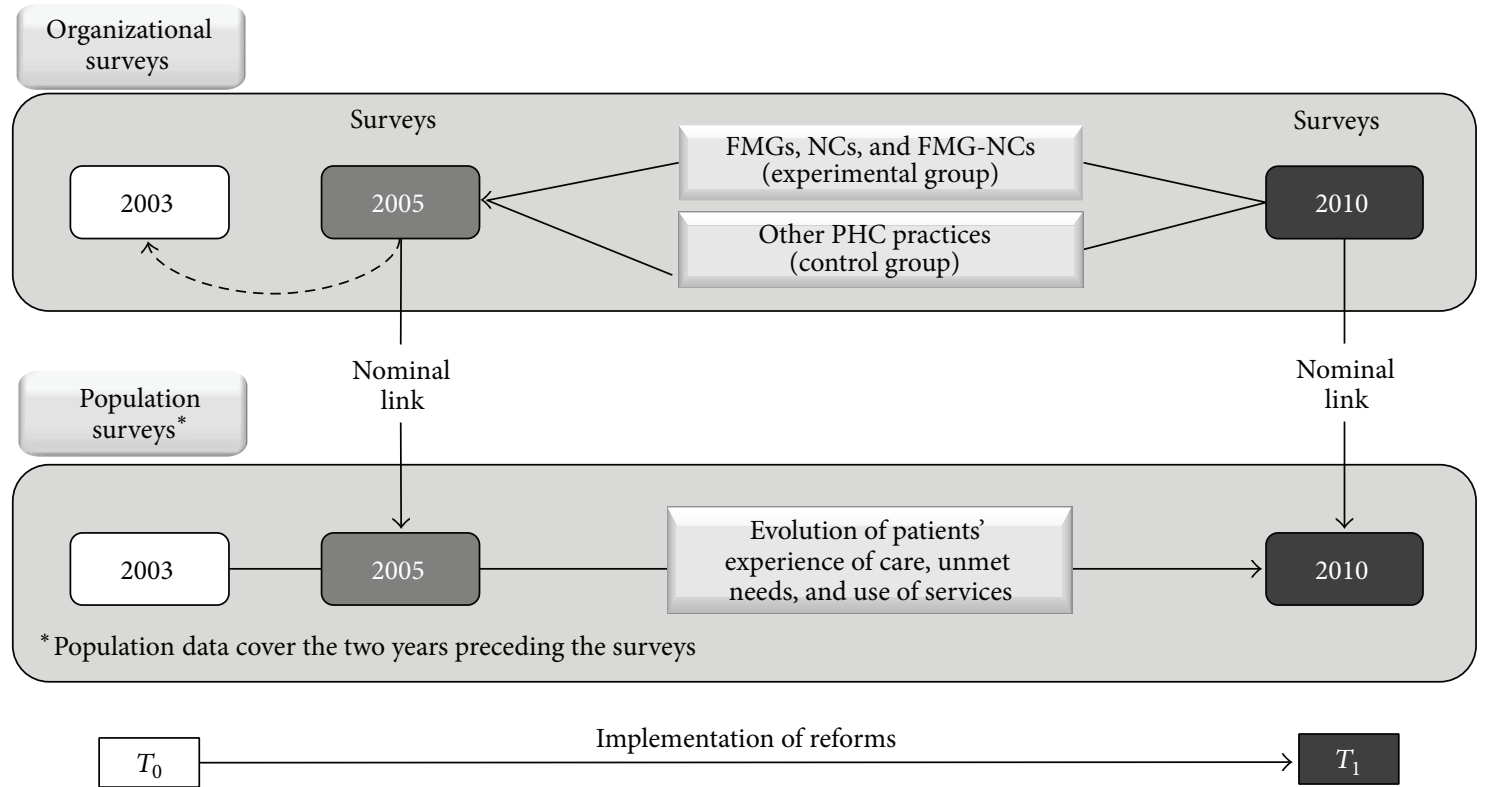

Figure 1: Study design.

walk-in services was gathered through telephone calls to the receptionists of all practices.

Population survey samples were stratified with approximatively 400 respondents in each of the 23 local territories regardless of their total population. The questionnaire was constructed drawing mainly on two validated instruments: the Primary Care Assessment Survey and the Primary Care Assessment Tool, to which we added questions when the topic had not been addressed [26-28]. It focused on respondents' attachment to a PHC practice, experience of care, and use of services in the two years preceding the surveys as well as unmet needs in the six months preceding the survey. The population questionnaire documented also individual characteristics such as gender, age, level of education, economic status, perceived health, and presence of morbidities [25].

Population surveys data were linked with information gathered on PHC organization through respondents' identification of their regular source of primary care in the two years preceding the survey (2003-2005 and 2008-2010). Failing to precisely identify a usual source of primary care, respondents identified the PHC practice most frequently attended in the two years preceding the survey. This practice was considered the respondent's usual source of care.

2.2. Organizational Variables. PHC organizations were classified into two groups: an experimental group that included new model practices (FMGs, NCs, and FMG-NCs) and a control group that included all other practices (Local Community Services Centres (LCSCs), Family Medicine Units (FMUs), and solo and group practices that were not FMG or NC). FMUs are academic training units that are likely to reflect current practice of family medicine and thus espouse hosting practices' dominant philosophy of care. For the purposes of this study, FMGs implemented in LCSC and FMU were included in the experimental group. These represented 11 LCSCs out of 49 and 9 FMUs out of 12 .

In 2010, among the different PHC organization models in Montréal and Montérégie, group and solo practices that were not FMG or NC were dominant, as they represented $73 \%$ of all organizations and were the usual source of care for $50 \%$ of all service users. Regardless of their status (FMG, NC, or none), they are privately run by physicians, who are paid on a fee-for-service basis. Costs are shared, but not income.

\subsection{Patients' Experience of Care, Use of Services, and Unmet} Needs. Information concerning patients' experience of care, use of services, and unmet needs was gathered from population surveys. Experience of care and utilization referred to the two years preceding the survey and unmet needs to the six months preceding the survey. Variables of experience of care were accessibility, continuity, comprehensiveness, responsiveness, and perceived care outcomes [29]. Operationalization details for these variables are presented in Table 8.

The 28 selected indicators of experience of care were grouped under five dimensions: accessibility (6 items), continuity (5 items), comprehensiveness (5 items), responsiveness (7 items), and perceived care outcomes (5 items). We carried out factor analysis within each of the five dimensions and calculated Cronbach's alpha which reached values of 0.60 or more for continuity (0.61), comprehensiveness (0.79), responsiveness (0.63), and care outcomes $(0.82)$ but was low for accessibility (0.30), presumably reflecting the formative nature of this index [30]. Accessibility items are not highly correlated. This characterizes a composite index rather than a reflective scale [31]. Variables of experience of care were expressed as scores on a 10-point scale. Utilization of services and unmet needs were dichotomous variables. 
2.4. Selection of Chronic Diseases. Data on chronic diseases come from population surveys. Respondents were asked if a doctor had told them they were suffering from specific chronic diseases. We then classified chronic diseases as follows: heart disease (coronary artery disease, heart failure), respiratory disease (chronic obstructive pulmonary disease, asthma), musculoskeletal disease (arthritis, osteoarthritis, and rheumatism), cardiometabolic diseases (hypertension, diabetes, and hypercholesterolemia), and other chronic diseases which was a residual and more heterogeneous category that included all remaining chronic diseases such as cancer, anemia, and gastrointestinal disorders. Mental illnesses were not excluded; they were considered as comorbidities associated with all the above categories, including the no chronic disease one; to be included in the broad category "with chronic disease," respondents had to have at least one chronic disease listed above. All others were included in the no chronic disease category.

2.5. Data Analysis. To test for differences in the evolution of experimental and control groups between 2003 and 2010, we applied the difference-in-differences (DD) technique and matched individuals with the propensity score method to ensure better comparability of the two groups [32-36]. This method is particularly well suited to compare change in outcomes over time between individuals exposed to an intervention (the experimental group) and individuals that were not (control group) [34]. Individuals were matched on the basis of propensity scores that estimate the probability for an individual with given characteristics to be attached to a PHC organization of the experimental group [37]. The propensity score acts as a balancing score that renders the distribution of observed baseline covariates similar between the experimental group and the control group [38]. The propensity score was calculated with logistic regression using sex, age, level of education, economic situation, immigration status, perception of health status, and morbidities as predictors.

The subjects were then matched applying the Kernel method, in which treated subjects are matched with a weighted average of all controls with weights that are inversely proportional to the distance between the propensity scores of treated individuals and controls. As the groups were very similar at the onset, nearly all subjects could be correctly matched. We carried out analyses using STATA (version 13). Difference-in-differences analyses were conducted separately on respondents with and without chronic diseases. Effect size, which expresses the magnitude of change, was measured in percentage and calculated by dividing DD scores by the average value of the indicators in the experimental and control groups at the baseline time period.

\section{Results}

3.1. Descriptive Results. Table 1 presents the percentage of respondents with a regular source of primary care. As expected, the percentage is higher for those with than for those without chronic diseases at both time periods. For both groups, the percentages significantly increased between 2003-2005 and 2008-2010.
TABLE 1: Percentage of respondents having a regular source of $\mathrm{PHC}$ according to the presence of chronic disease, 2003-2005 and 20082010 .

\begin{tabular}{lccc}
\hline & $2003-2005$ & $2008-2010$ & $p^{*}$ \\
\hline $\begin{array}{l}\text { At least one } \\
\text { chronic disease }\end{array}$ & $(n=4870)$ & $(n=5424)$ & \\
\hline $\begin{array}{l}\text { No chronic } \\
\text { disease }\end{array}$ & $(n=4336)$ & $(n=3756)$ & $<0.001$ \\
\hline
\end{tabular}

${ }^{*}$ Difference of proportions test.

TABle 2: Percentage of users with various chronic diseases, 20032005 and 2008-2010.

\begin{tabular}{lccc}
\hline & $\begin{array}{c}2003-2005 \\
(n=6198)\end{array}$ & $\begin{array}{c}2008-2010 \\
(n=6753)\end{array}$ & $p^{*}$ \\
\hline At least one chronic disease & 58.9 & 64.4 & $<0.001$ \\
Cardiac & 7.4 & 9.4 & $<0.001$ \\
Respiratory & 14.6 & 15.3 & 0.287 \\
Musculoskeletal & 23.4 & 26.0 & $<0.001$ \\
Cardiometabolic & 24.0 & 33.4 & $<0.001$ \\
Other chronic diseases & 31.2 & 25.9 & $<0.001$ \\
\hline
\end{tabular}

${ }^{*}$ Difference of proportions test.

Table 2 shows, among those who had a regular source of primary care, percentages of users with different chronic diseases in the 2005 and 2010 surveys.

Table 3 presents for each category of chronic diseases in the two time periods the percentage of those who, aside from that disease, also present other chronic morbidities. Cardiac and musculoskeletal diseases have a higher percentage of associated multiple chronic morbidities. Data for the two time periods are generally comparable except for "cardiometabolic" and "other chronic diseases."

Tables 4 and 5 present data on other users' characteristics for differences between control and experimental groups of the two samples. There are very few differences that are statistically significant.

3.2. Evolution of Experience of Care, Use of Services, and Unmet Needs. The impact of the introduction of new PHC models for individuals with chronic diseases was tested by comparing, in 2003-2005 and 2008-2010, individuals attached to FMG-NC, FMG, and NC (experimental group) to those attached to other practices that had not changed (control group) with regard to experience of care, unmet needs, and use of services. As shown in Table 6, accessibility presents the lowest score among indices of experience of care. It decreased in both control and experimental groups over the years, but the experimental group remained with higher score and statistically significant differences at both times $(p=$ 0.048; $p=0.004)$. However, the change was not sufficient to yield a significant DD value $(p=0.640)$. Continuity was high in both groups. At both times, scores were higher for the control group, and the difference even increased in 2008$2010(p=0.001 ; p<0.001)$. The DD value approached the 0.05 level of significance $(p=0.08)$ with a small negative 


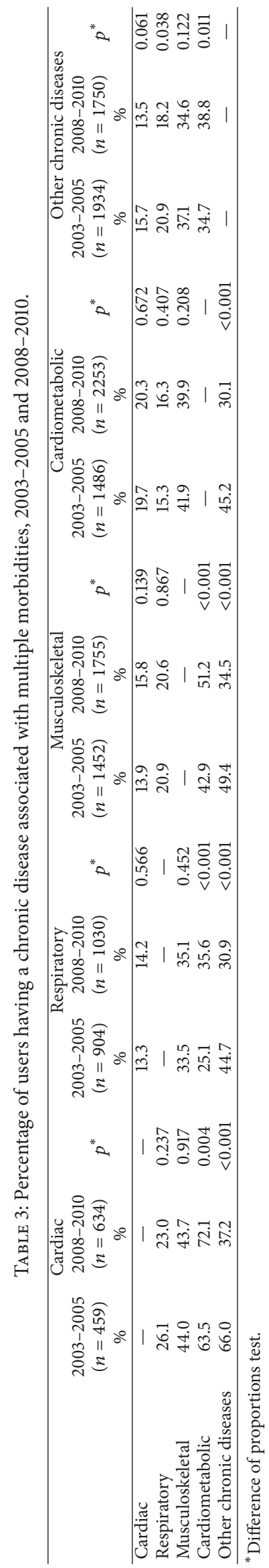


TABLE 4: Percentage (\%) of users having at least one chronic disease according to selected characteristics, 2003-2005 and $2008-2010$.

\begin{tabular}{|c|c|c|c|c|c|c|c|}
\hline & & & 2003-2005 & & & $2008-2010$ & \\
\hline & & $\begin{array}{c}\text { Control } \\
(n=1831)\end{array}$ & $\begin{array}{c}\text { Experimental } \\
\quad(n=1770)\end{array}$ & $p^{*}$ & $\begin{array}{c}\text { Control } \\
(n=2198)\end{array}$ & $\begin{array}{c}\text { Experimental } \\
\quad(n=2151)\end{array}$ & $p^{*}$ \\
\hline Sex & Male & 33.8 & 34.3 & 0.732 & 39.1 & 39.2 & 0.916 \\
\hline Sex & Female & 66.2 & 65.7 & & 60.9 & 60.8 & \\
\hline & $18-29$ & 11.3 & 9.3 & 0.050 & 4.7 & 5.0 & 0.709 \\
\hline Age & $30-44$ & 21.0 & 23.2 & 0.113 & 13.6 & 16.5 & 0.007 \\
\hline Mge & $45-64$ & 43.5 & 42.8 & 0.645 & 47.5 & 46.4 & 0.505 \\
\hline & 65 or more & 24.2 & 24.8 & 0.700 & 34.3 & 32.1 & 0.135 \\
\hline & No diploma & 19.5 & 23.3 & 0.005 & 18.5 & 21.4 & 0.018 \\
\hline Level of education & High school diploma & 32.7 & 34.9 & 0.174 & 31.3 & 32.1 & 0.538 \\
\hline Lever or equedion & Collegial diploma & 24.2 & 22.0 & 0.115 & 19.6 & 19.7 & 0.932 \\
\hline & University diploma & 23.5 & 19.8 & 0.006 & 30.7 & 26.8 & 0.005 \\
\hline & Lowest & 13.4 & 12.7 & 0.518 & 12.2 & 12.0 & 0.841 \\
\hline Economic status & Medium-low & 32.1 & 34.7 & 0.101 & 31.8 & 31.8 & 0.975 \\
\hline & Medium-high & 29.8 & 30.0 & 0.878 & 33.7 & 35.7 & 0.158 \\
\hline & Highest & 24.7 & 22.7 & 0.141 & 22.3 & 20.5 & 0.130 \\
\hline & Born in Canada & 84.2 & 91.2 & $<0.001$ & 84.1 & 88.8 & $<0.001$ \\
\hline Immigration status & Imm. $<10$ years & 2.8 & 1.5 & 0.018 & 1.7 & 2.3 & 0.131 \\
\hline & Imm. $\geq 10$ years & 13.1 & 7.3 & $<0.001$ & 14.2 & 8.9 & $<0.001$ \\
\hline & Bad/average & 25.2 & 26.6 & 0.346 & 23.7 & 23.2 & 0.695 \\
\hline Perceived health & Good & 34.2 & 33.9 & 0.854 & 33.7 & 34.4 & 0.632 \\
\hline & Very good & 28.9 & 28.3 & 0.671 & 31.2 & 30.1 & 0.457 \\
\hline & Excellent & 11.7 & 11.2 & 0.676 & 11.5 & 12.3 & 0.384 \\
\hline & Cardiac & 12.8 & 12.7 & 0.951 & 13.0 & 16.2 & 0.002 \\
\hline & Respiratory & 24.0 & 26.2 & 0.131 & 24.4 & 22.9 & 0.241 \\
\hline Chronic disease & Musculoskeletal & 39.3 & 41.4 & 0.214 & 39.6 & 41.1 & 0.323 \\
\hline & Cardiometabolic & 41.9 & 40.6 & 0.401 & 52.7 & 50.9 & 0.241 \\
\hline & Other chronic diseases & 53.3 & 54.1 & 0.622 & 39.8 & 40.7 & 0.518 \\
\hline
\end{tabular}

${ }^{*}$ Difference of proportions test.

effect size of $-1.5 \%$. The pattern for comprehensiveness and responsiveness is very similar to that of continuity with nonsignificant DD values. Care outcomes show higher scores for the control group with increased differences in 2008-2010. This resulted in a significant DD value of -1.6 in favor of the control group $(p=0.044)$ and an effect size of $-1.8 \%$. For unmet needs and utilization indicators, the results show no significant results except for high utilization of the usual source of primary care that decreased in both groups, but more so in the experimental group (-7.9) than in the control group $(-4.0)$, resulting in a significant and negative DD value ( $p=0.049)$, corresponding to a $12.7 \%$ decrease.

We carried out similar analyses for respondents with no chronic disease. Data presented in Table 7 show overall decline in accessibility over time in both experimental and control groups. However, the decline was larger in the control than in the experimental group while it was very small in the experimental group, yielding a positive and significant DD value ( $p<0.001)$ with a $5.5 \%$ increase. As regards continuity, the differences between groups remain constant over time in favor of the control group and this lack of change resulted in a nonsignificant $\mathrm{DD}$ value $(p=0.563)$. The difference between groups for responsiveness turned in favor of the experimental group in 2008-2010, resulting in a positive and significant DD value ( $p=0.007)$ with a positive $2.3 \%$ change. Aside from these results, the evolution in both groups for all other indicators has been comparable, with no significant DD values.

\section{Discussion}

Patients with chronic diseases in the experimental group had a worse experience of care than those in the control group in 2008-2010 on all indicators, except accessibility that was better and responsiveness that showed no difference. The percentage of users who reported unmet needs was lower in the control group and hospitalization was slightly higher in the experimental group, but these differences were not significant.

Looking at the evolution of experience of care for patients with chronic diseases in the two groups, measured by DD values, we observed that continuity increased more in the experimental than in the control group although the DD value failed to reach the 0.05 level of significance $(p=0.081)$. 
TABLE 5: Percentage (\%) of users without a chronic disease according to selected characteristics, 2003-2005 and $2008-2010$.

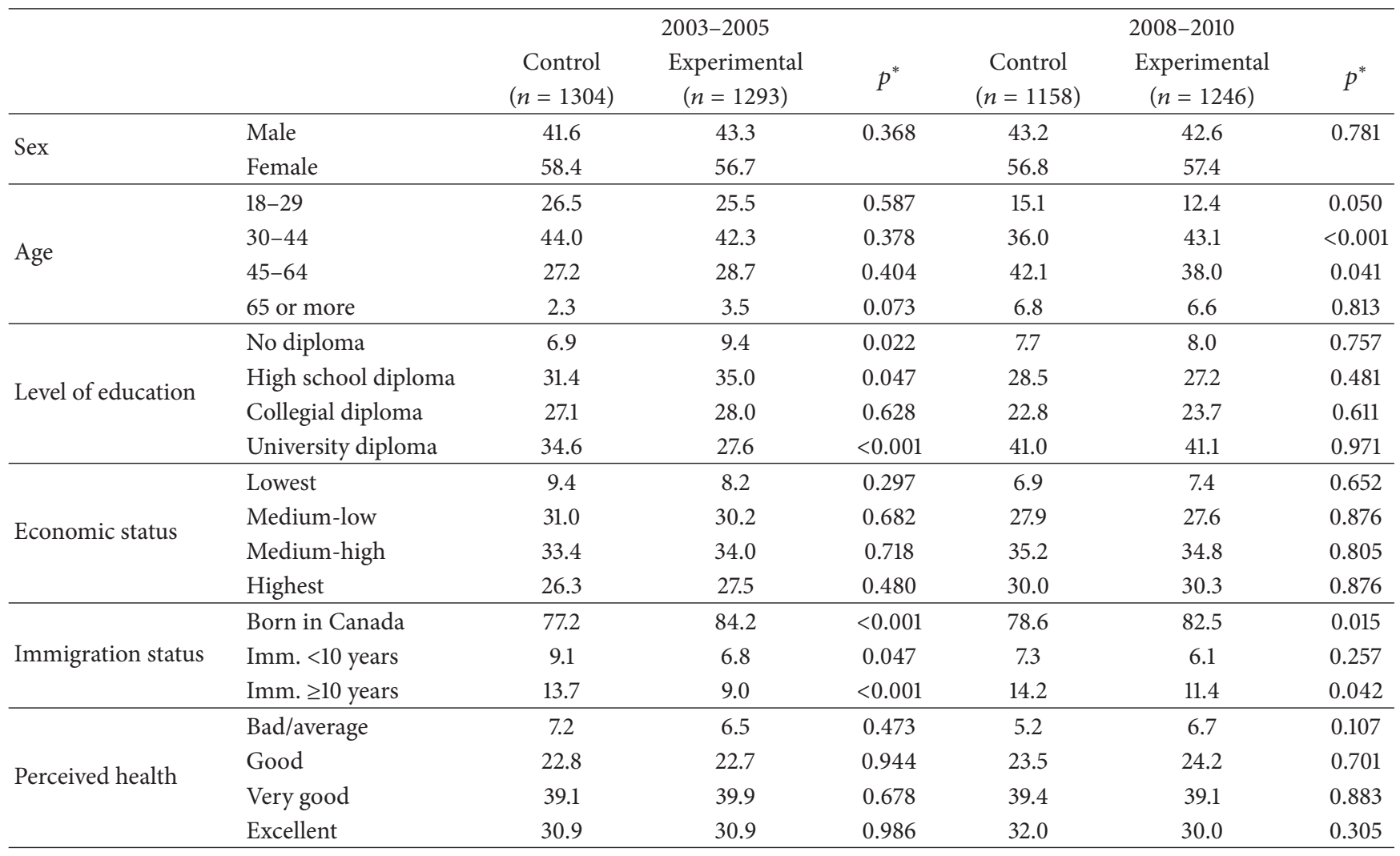

${ }^{*}$ Difference of proportions test.

Perceived care outcomes increased more in the control than in the experimental group resulting in a negative and significant DD value. The percentage of users attending more often their usual source of primary care also declined more in the experimental than in the control group and these results are statistically significant $(p=0.049)$ with a percentage of change close to $13 \%$.

The findings for patients with no chronic disease contrast with those of patients with chronic diseases. In 2008-2010, accessibility was better in the experimental group than in control group $(p<0.001)$, but continuity was worse $(p<$ $0.001)$. There was no other significant result. DD values are positive and statistically significant for accessibility with a 5\% effect size, indicating less unfavorable evolution for the experimental group $(p<0.001)$. Another significant and positive $\mathrm{DD}$ value is for responsiveness $(p=0.007)$. Continuity shows no difference between groups in its evolution, nor with all the other indicators of experience of care, unmet needs, and utilization.

These findings raise concern about expected benefits resulting from Quebec healthcare reforms. First, it is disappointing to see that while continuity improved in the population it did so more in the control than in the experimental groups. The main objective pursued by the reform in providing additional resources to new model practices was precisely to improve continuity of care, especially among individuals with chronic diseases [11, 12]. A possible explanation for this result could be that financial incentives to enrollment of patients with chronic diseases were extended to all PHC organizations, thus offsetting the advantage enjoyed up to that point by FMGs. Another explanation may be increased provision of services by nurses in FMGs and NCs that are not included in our measures of continuity. Our measures of experience of care targeted mainly services rendered by physicians. Consequently, they may underestimate services rendered by professionals other than doctors.

These findings regarding continuity do not support those of an earlier study conducted by Tourigny et al. among 1,275 patients followed up in five FMGs [39]. That study found an increase in continuity among patients 18 months after their enrollment in FMGs. However, Tourigny et al's study did not have a control group and was limited to only five FMGs.

A second unanticipated result concerns accessibility which showed less unfavorable evolution for patients without chronic diseases in the experimental group than for patients with chronic diseases. Again, the establishment of new models of PHC organizations, and particularly of NCs, was aimed at improving accessibility to care for the general population, but in priority to the neediest individuals. These included patients with chronic diseases. It is rather ironic that the ones more in need were more affected by the unfavorable evolution of accessibility.

Another finding that raises concerns is the lack of impact of the reform on unmet needs and use of services. Conversely, the larger decrease in the percentage of the high users of services at the usual source of primary care in the experimental 


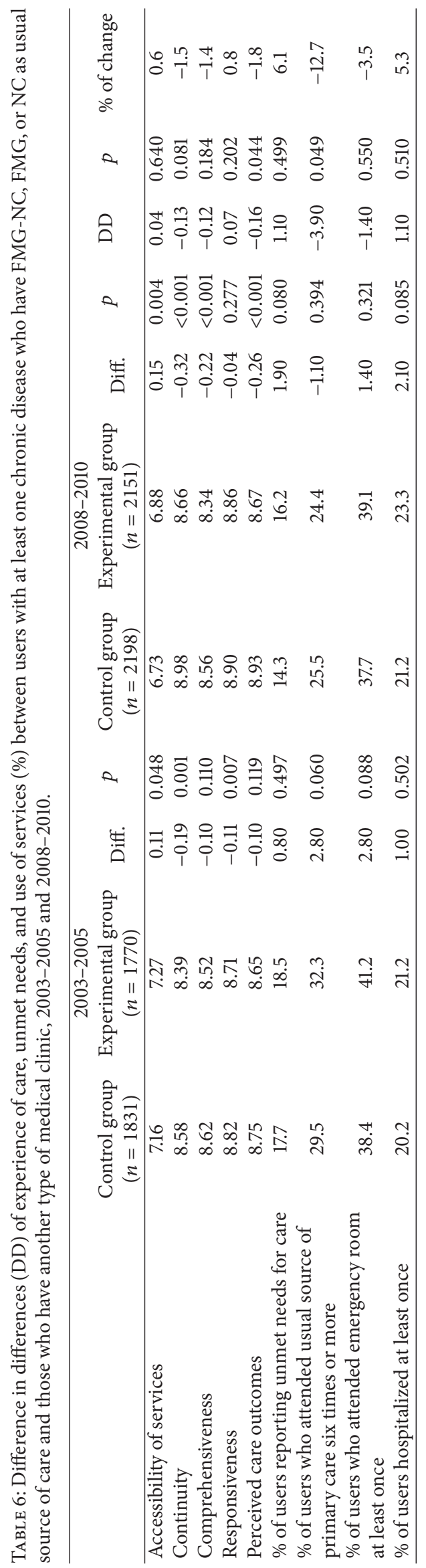




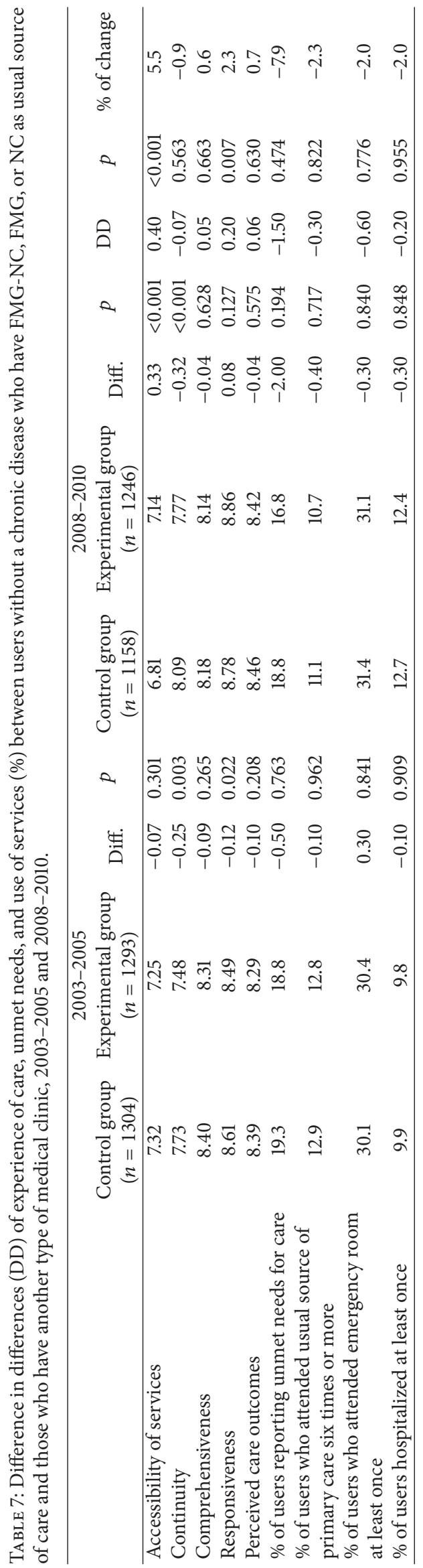




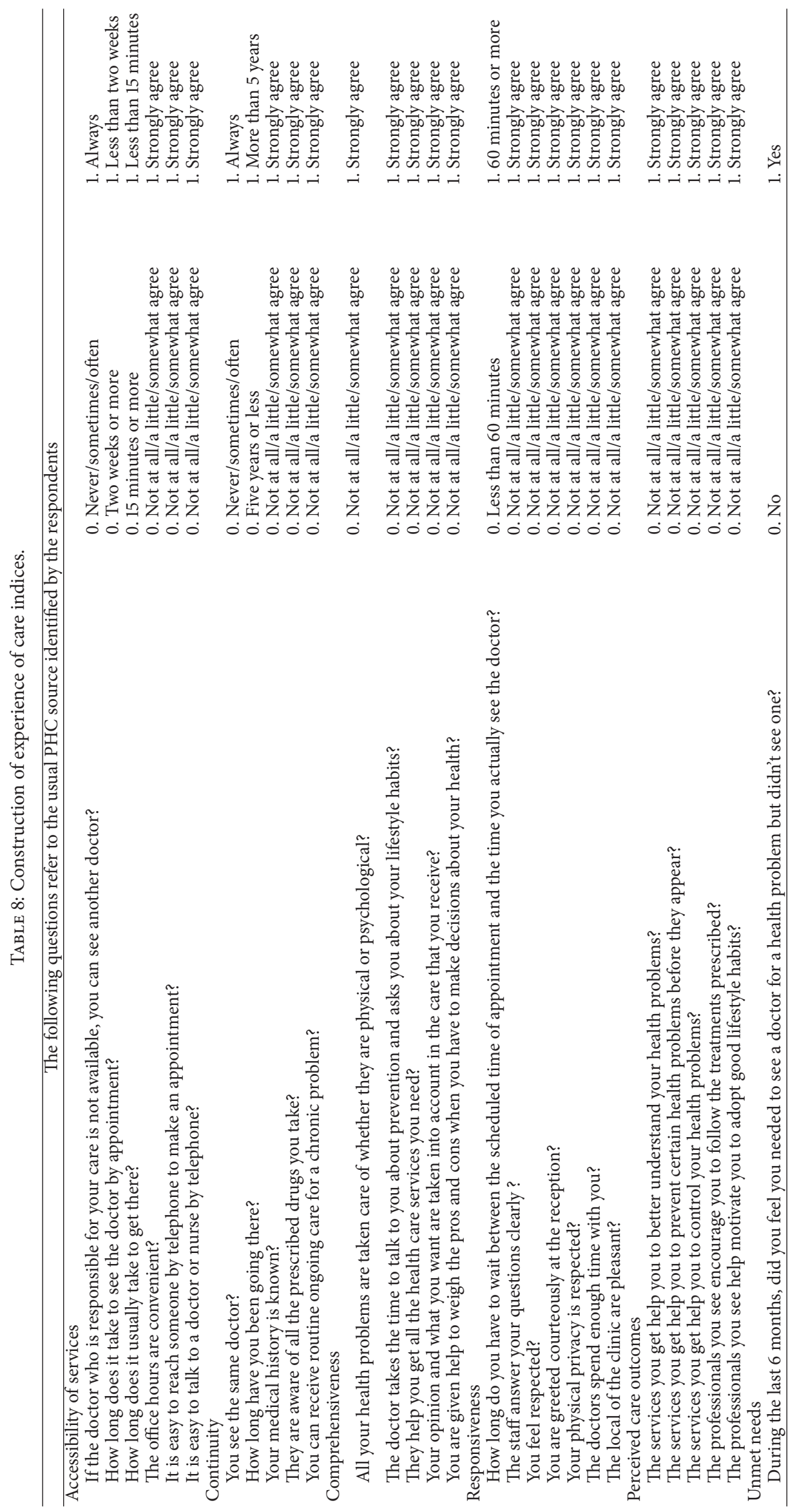


group can be considered as a positive outcome, possibly associated with more comprehensive and preventive care. It could also result from substitution between services provided by physicians and those provided by nurses or other health professionals. We had no data to support these plausible explanations. However, the impact of this outcome remains marginal if not coupled to decrease in ER attendance and hospitalization that represent the most costly services.

Our findings regarding accessibility and utilization corroborate studies reporting on FMGs. In an ecological study of the entire Quebec population, Dunkley-Hickin found that a higher degree of physicians' participation in FGM in a geographical area was not associated with improved accessibility among users of services living in that area [40].

In a large scale cohort study involving 79 FMGs, Strumpf observed no improvement in accessibility but a slight reduction in use of primary care and specialist services [41]. Based on the same administrative data banks, Héroux et al. followed up a cohort of 122,722 patients enrolled in FMGs compared to 675,102 who were not [42]. They found a small reduction in number of emergency visits for those attached to FMGs, but, as in our study, no change in hospitalization.

None of those studies has reported specifically on patients with chronic diseases compared to those without chronic diseases. In an earlier study conducted on the 2005 sample, we explored the relationship between models of PHC organizations and experience of care. We concluded that the "coordination-integrated model," which matched the attributes of the ideal type of FMGs that were to be implemented at that time, emerged as the model with the greatest potential to concomitantly achieve high levels of accessibility and continuity for patients with chronic diseases [13]. Obviously, this prediction has not yet come true. It could be that the reform had an effect on both PHC organizations that have become FMGs or NCs and those that have not changed their status. It was not possible with our data to test that spillover effect hypothesis.

According to Rothman and Wagner, the future of primary healthcare largely depends on ability to improve healthcare delivery to meet the needs of chronically ill patients [3]. The Chronic Care Model provides six interrelated components for achieving such change among which is delivery system redesign [4]. This last condition does not seem to have been met in the implementation of new PHC models in Quebec.

A recent report of the Auditor General of Quebec to the National Assembly casts a critical look at the implantation of FMGs and NCs [43]. It explains the relative failure of these PHC organizations to fully attain the objectives established by the Ministry of Health and Social Services by many factors, notably the absence of clear rules, guidelines, and incentives and, above all, the lack of control of the Ministry and the Regional Agencies on the process of implementation. This means that several new $\mathrm{PHC}$ practices received complementary funding without respecting all the components of the contractual engagement.

Along the same lines, a recent report of the C.D. Howe Institute underlines that important factors associated with the slow implementation of FMGs are the low registration rate of patients and the deficient development of teamwork
[44]. The findings of our study concur with those of other studies and support concerns expressed by analysts.

\section{Limitations}

Our study has limitations. The use of difference-in-differences (DD) method for isolating the specific effect of the reform is fully justified because it provides good internal validity. However, when carried out in a complex system such as the healthcare system, DD analyses cannot completely rule out the possibility of spillover effects. For example, it is possible that some aspects of the healthcare policies spilled over and influenced PHC practices of the unexposed control group or that new PHC model practices exerted a mimetic influence on the other practices [45].

Surveys lend themselves to possible recall bias by respondents. Concerning experience of care, unmet needs, and use of services, if present, biases should be equally distributed among respondents. Recall biases are less likely to occur when reporting events extending over a certain period of time rather than single events taking place at a given point in time.

Another limitation is self-reporting of chronic morbidities. Responses are always limited by respondents' subjective understanding and their capacity to report accurately medical information. The wording of the questions attenuated this possible effect by referring to validation of the diagnosis by a doctor (i.e., "has a doctor ever told you that you have diabetes?"). Categories of chronic diseases were defined and operationalized in the same way in both 2005 and 2010 surveys. However, for the category "other chronic diseases," it is possible that our redefinition and classification were more inclusive in 2005, which may explain the higher percentage of respondents reporting this type of morbidity in 2005.

As pointed out in Methods, mental illnesses were not excluded but left in all categories of physical chronic conditions, including the no disease category. Many reasons justified our decision. The questions concerning mental health problems were slightly different in 2005 and 2010 as well as the time period reference: "have you ever had..." in 2005 and "in the last 2 years, did you see your doctor..." in 2010. This may explain the higher percentage of individuals reporting mental health problems in 2005 than in 2010. Aside from this difference between the two surveys, mental health problems happened to be evenly distributed among our categories of chronic physical diseases, including the no disease category. Consequently, we thought that their inclusion would minimize potential biases while increasing statistical power.

Measures of experience of care reflect patients' point of view and perceptions. They are subjective but still most appropriate to use in a patient-centered perspective. Our questionnaire largely draws from two validated instruments (PCAS and PCAT) [26-28]. We constructed new scales of experience of care, more adapted to our context. Following the classical theory of measurement, we carried out factor analysis and calculated Cronbach's alpha coefficients which have values close to the commonly accepted level (0.70) except for accessibility (0.30) [46]. We still considered accessibility a valid measure based on the formative approach to 
measurement. In contrast to the classical reflective approach, in the formative approach items composing an index are not necessarily correlated or substitutable with each other, as is the case in reflective scales [30, 31].

As mentioned earlier, our scales of experience of care may fail to include processes of care rendered by health professionals other than doctors. In that sense, they may underestimate experience of care in contexts of multidisciplinary work and thus fail to fully account for the effect of introducing new types of professionals, as is the case in FMGs and NCs. The possibility of such a bias has been suggested, particularly by researchers using large administrative data banks based on physicians' reimbursement claims $[47,48]$.

The 2003-2005 baseline time period was very close to the onset of the reform. The first FMGs were accredited in March 2003. Since questions related to experience of care and use of services referred to the two years preceding the population surveys, we are confident that the 2003-2005 time period was a valid baseline in the context of a natural experiment. Furthermore, as expected, changes took some time before being fully implemented in PHC practices after their accreditation.

\section{Conclusion}

Our study's findings raise questions concerning benefits resulting from the introduction of new $\mathrm{PHC}$ practice models in Québec. The aim of the PHC reform was mainly to improve accessibility and continuity of care for the entire population, but especially for individuals in greater needs such as those with chronic diseases. Our findings show that, for patients with chronic diseases attached to new PHC model practices, continuity increased less than for those attached to other practices. Accessibility decreased for all users of services but much less for those with no chronic disease in the new model PHC practices but did not change for those with chronic diseases. These results raise equity concerns since the ones with more needs seem to have benefitted less from the reform than those less in needs. Furthermore, the reform did not reduce the use of the most costly services, ER attendance, and hospitalization, nor did it reduce the reporting of unmet needs. Based on those findings, it is difficult to conclude that the Québec healthcare reform has been so far successful.

\section{Additional Points}

Experience of care was not better in 2010 among patients with chronic diseases in new PHC practices models. Evolution of experience of care was not more favorable among these patients than those in the other practices. For patients with no chronic disease and those attached to the new PHC practice models, accessibility increased more than for patients with chronic diseases. So far, the benefits resulting from the reform have been less than expected.

\section{Competing Interests}

The authors declare that they have no competing interests.

\section{References}

[1] A.-M. Broemeling, D. E. Watson, and F. Prebtani, "Population patterns of chronic health conditions, co-morbidity and healthcare use in Canada: implications for policy and practice," Healthcare Quarterly, vol. 11, no. 3, pp. 70-76, 2008.

[2] E. H. Wagner, B. T. Austin, C. Davis, M. Hindmarsh, J. Schaefer, and A. Bonomi, "Improving chronic illness care: translating evidence into action," Health Affairs, vol. 20, no. 6, pp. 64-78, 2001.

[3] A. A. Rothman and E. H. Wagner, "Chronic illness management: what is the role of primary care?" Annals of Internal Medicine, vol. 138, no. 3, pp. 256-261, 2003.

[4] T. Bodenheimer, E. H. Wagner, and K. Grumbach, "Improving primary care for patients with chronic illness," The Journal of the American Medical Association, vol. 288, no. 14, pp. 1775-1779, 2002.

[5] K. Davis, S. C. Schoenbaum, and A.-M. Audet, "A 2020 vision of patient-centered primary care," Journal of General Internal Medicine, vol. 20, no. 10, pp. 953-957, 2005.

[6] A. Scobie, N. J. MacKinnon, S. Higgins, H. Etchegary, and R. Church, "The medical home in Canada: patient perceptions of quality and safety," Healthcare Management Forum, vol. 22, no. 1, pp. 47-51, 2009.

[7] M. Mirzaei, C. Aspin, B. Essue et al., "A patient-centred approach to health service delivery: improving health outcomes for people with chronic illness," BMC Health Services Research, vol. 13, article 251, 2013.

[8] L. Nasmith, P. Ballem, R. Baxter et al., Transforming Care for Canadians with Chronic Health Conditions: Put People First, Expect the Best, Manage for Results, Canadian Academy of Health Sciences, Ottawa, Canada, 2010.

[9] The College of Family Physicians of Canada, A Vision for Canada: Family Practice-The Patient's Medical Home, Mississauga, Canada, 2011.

[10] B. Starfield, Primary Care: Balancing Health Needs, Services and Technology, Oxford University Press, New York, NY, USA, 1998.

[11] M. P. Pomey, E. Martin, and P. G. Forest, "Québec's family medicine groups: innovation and compromise in the reform of front-line care," Canadian Political Science Review, vol. 3, no. 4, pp. 31-46, 2009.

[12] M. Breton, J. F. Levesque, R. Pineault, and W. Hogg, "Primary care reform: can Québec's family medicine group model benefit from the experience of Ontario's family health teams?" Healthcare Policy, vol. 7, no. 2, pp. e122-e135, 2011.

[13] R. Pineault, S. Provost, M. Hamel, A. Couture, and J. F. Levesque, "The influence of primary health care organizational models on patients' experience of care in different chronic disease situations," Chronic Diseases in Canada, vol. 31, no. 3, pp. 109-120, 2011.

[14] M. W. Friedberg, K. L. Coltin, D. G. Safran, M. Dresser, A. M. Zaslavsky, and E. C. Schneider, "Associations between structural capabilities of primary care practices and performance on selected quality measures," Annals of Internal Medicine, vol. 151, no. 7, pp. 456-463, 2009.

[15] P. J. O’Connor, J. M. Sperl-Hillen, N. P. Pronk, and T. Murray, "Primary care clinic-based chronic disease care: features of successful programs," Disease Management and Health Outcomes, vol. 9, no. 12, pp. 691-698, 2001.

[16] S. M. Shortell, R. Gillies, J. Siddique et al., "Improving chronic illness care: a longitudinal cohort analysis of large physician organizations," Medical Care, vol. 47, no. 9, pp. 932-939, 2009. 
[17] L. P. Casalino, M. F. Pesko, A. M. Ryan et al., "Small primary care physician practices have low rates of preventable hospital admissions," Health Affairs, vol. 33, no. 9, pp. 1680-1688, 2014.

[18] A. C. Tsai, S. C. Morton, C. M. Mangione, and E. B. Keeler, "A meta-analysis of interventions to improve care for chronic illnesses," The American Journal of Managed Care, vol. 11, no. 8, pp. 478-488, 2005.

[19] P. A. Nutting, W. P. Dickinson, L. M. Dickinson et al., "Use of Chronic Care model elements is associated with higher-quality care for diabetes," Annals of Family Medicine, vol. 5, no. 1, pp. 14-20, 2007.

[20] M. L. Pearson, S. Wu, J. Schaefer et al., "Assessing the implementation of the chronic care model in quality improvement collaboratives," Health Services Research, vol. 40, no. 4, pp. 978996, 2005.

[21] S. Cretin, S. M. Shortell, and E. B. Keeler, "An evaluation of collaborative interventions to improve chronic illness care," Evaluation Review, vol. 28, no. 1, pp. 28-51, 2004.

[22] G. M. Russell, S. Dahrouge, W. Hogg, R. Geneau, R. Muldoon, and M. Tuna, "Managing chronic disease in Ontario primary care: the impacts of organizational factors," The Annals of Family Medicine, vol. 7, no. 4, pp. 309-318, 2009.

[23] R. Pineault, J. F. Levesque, D. Roberge, M. Hamel, P. Lamarche, and J. Haggerty, Accessibility and Continuity of Care: A Study of Primary Healthcare in Québec, The Canadian Institutes of Health Research and the Canadian Health Services Research Foundation, Ottawa, Canada, 2008.

[24] J.-F. Lévesque, D. E. Feldman, V. Lemieux, A. Tourigny, J.-P. Lavoie, and P. Tousignant, "Variations in patients' assessment of chronic illness care across organizational models of primary health care: a multilevel cohort analysis," Healthcare Policy, vol. 8, no. 2, pp. e108-e123, 2012.

[25] J.-F. Levesque, R. Pineault, S. Provost et al., "Assessing the evolution of primary healthcare organizations and their performance (2005-2010) in two regions of Québec province: Montréal and Montérégie," BMC Family Practice, vol. 11, article 95, 2010.

[26] L. Shi, B. Starfield, and J. Xu, "Validating the adult primary care assessment tool," The Journal of Family Practice, vol. 50, no. 2, pp. 161-175, 2001.

[27] D. G. Safran, M. Kosinski, A. R. Tarlov et al., "The primary care assessment survey: tests of data quality and measurement performance," Medical Care, vol. 36, no. 5, pp. 728-739, 1998.

[28] J. Haggerty, R. Pineault, M. D. Beaulieu et al., Accessibility and Continuity of Primary Care in Québec, The Canadian Health Services Research Foundation, Ottawa, Canada, 2004.

[29] D. G. Safran, "Defining the future of primary care: what can we learn from patients?” Annals of Internal Medicine, vol. 138, no. 3, pp. 248-255, 2003.

[30] K. A. Bollen and S. Bauldry, "Three Cs in measurement models: causal indicators, composite indicators, and covariates," Psychological Methods, vol. 16, no. 3, pp. 265-284, 2011.

[31] A. Diamantopoulos and H. M. Winklhofer, "Index construction with formative indicators: an alternative to scale development," Journal of Marketing Research, vol. 38, no. 2, pp. 269-277, 2001.

[32] J. D. Angrist and J. S. Pischke, "Parallel words: fixed effects, difference-in-differences, and panel date," in Mostly Harmless Econometric, pp. 221-246, Princeton University Press, Princeton, NJ, USA, 2009.

[33] B. D. Meyer, "Natural and quasi-experiments in economics," Journal of Business \& Economic Statistics, vol. 13, no. 2, pp. 151161, 1995.
[34] P. J. Gertler, S. Martinez, P. Premand, L. B. Rawlings, and C. M. J. Vermeersch, "Difference-in-differences," in Impact Evaluation in Practice, P. J. Gertler, S. Martinez, P. Premand, L. B. Rawlings, and C. M. J. Vermeersch, Eds., pp. 95-105, International Bank for Reconstruction and Development/The World Bank, Washington, DC, USA.

[35] B. A. Griffin and D. F. McCaffrey, "Nonparametric matching and propensity scores," in Encyclopedia of Health Economics, A. J. Culyer, Ed., pp. 370-374, Elsevier, Oxford, UK, 1st edition, 2014.

[36] J. B. Dimick and A. M. Ryan, "Methods for evaluating changes in health care policy: the difference-in-differences approach," The Journal of the American Medical Association, vol. 312, no. 22, pp. 2401-2402, 2014.

[37] E. A. Stuart, "Matching methods for causal inference: a review and a look forward," Statistical Science, vol. 25, no. 1, pp. 1-21, 2010.

[38] P. C. Austin, "An introduction to propensity score methods for reducing the effects of confounding in observational studies," Multivariate Behavioral Research, vol. 46, no. 3, pp. 399-424, 2011.

[39] A. Tourigny, M. Aubin, J. Haggerty et al., "Patients' perceptions of the quality of care after primary care reform: family medicine groups in Quebec," Canadian Family Physician, vol. 56, no. 7, pp. e273-e282, 2010.

[40] C. Dunkley-Hickin, Effects of primary care reform in Quebec on access to primary health care services [M.S. thesis], McGill University, Department of Epidemiology and Biostatistics, 2013.

[41] E. Strumpf, "L'évaluation des groupes de médecine de famille (GMF) [oral presentation]. L'évaluation économique et la recherche sur les services de santé," in Proceedings of the Seminar Organized by the Ministère des Affaires Sociales, de la Santé et des Droits des Femmes, Paris, France, December 2014.

[42] J. Héroux, E. E. M. Moodie, E. Strumpf, N. Coyle, P. Tousignant, and M. Diop, "Marginal structural models for skewed outcomes: identifying causal relationships in health care utilization," Statistics in Medicine, vol. 33, no. 7, pp. 1205-1221, 2014.

[43] Auditor General of Quebec, "Groupe de médecine de famille et cliniques-réseau, services de première ligne dans le réseau de la santé et des services sociaux," in Rapport du Vérificateur Général du Québec à l'Assemblée Nationale Pour L'année 20152016. Vérification De Loptimisation des Ressources Services de Première Ligne Dans le Réseau de la Santé et des Services Sociaux, chapter 5, Gouvernement du Québec, Québec, Canada, 2015.

[44] C. Forget, "The case of the vanishing Quebec physicians: how improve access to care," Commentary 410, C.D. Howe Institute, 2014.

[45] R. Pineault, R. Borgès Da Silva, A. Prud'Homme et al., "Impact of Québec's healthcare reforms on the organization of primary healthcare (PHC): a 2003-2010 follow-up," BMC Health Services Research, vol. 14, article 229, 2014.

[46] J. Bland and D. Altman, "Statistical notes: Cronbach's alpha," British Medical Journal, vol. 314, no. 7082, article 572, 1997.

[47] P. Tousignant, M. Diop, M. Fournier et al., "Validation of 2 new measures of continuity of care based on year-to-year followup with known providers of health care," Annals of Family Medicine, vol. 12, no. 6, pp. 559-567, 2014.

[48] S. Provost, J. Pérez, R. Pineault, R. Borgès Da Silva, and P. Tousignant, "An algorithm using administrative data to identify patient attachment to a family physician," International Journal of Family Medicine, vol. 2015, Article ID 967230, 11 pages, 2015. 


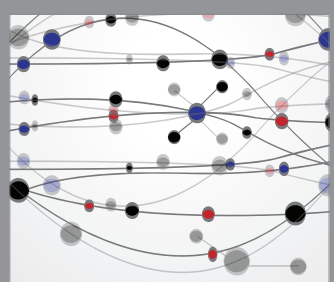

The Scientific World Journal
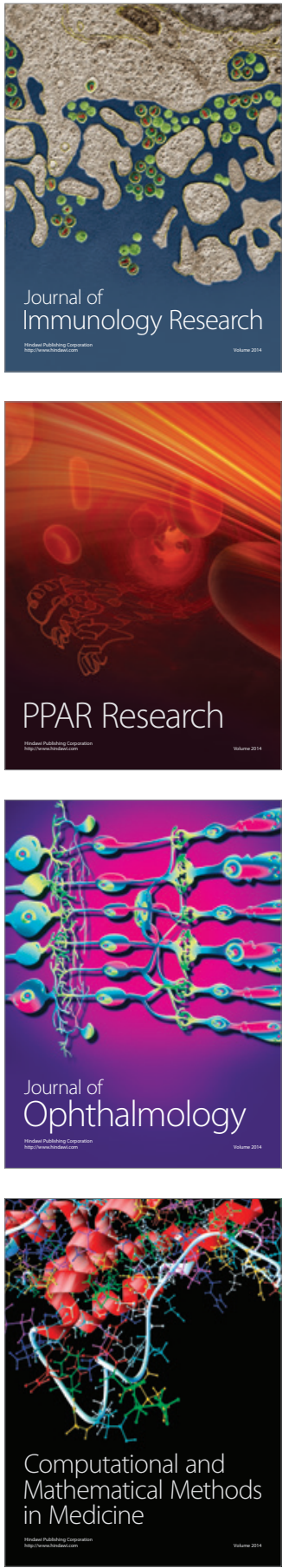

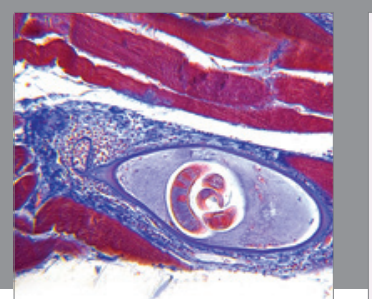

Gastroenterology Research and Practice

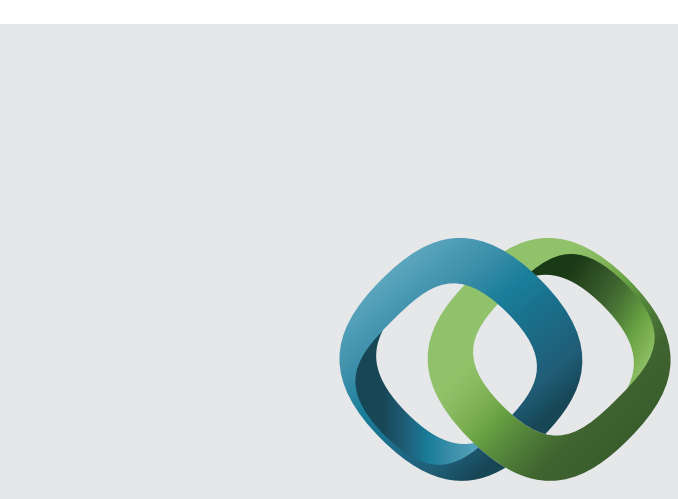

\section{Hindawi}

Submit your manuscripts at

http://www.hindawi.com
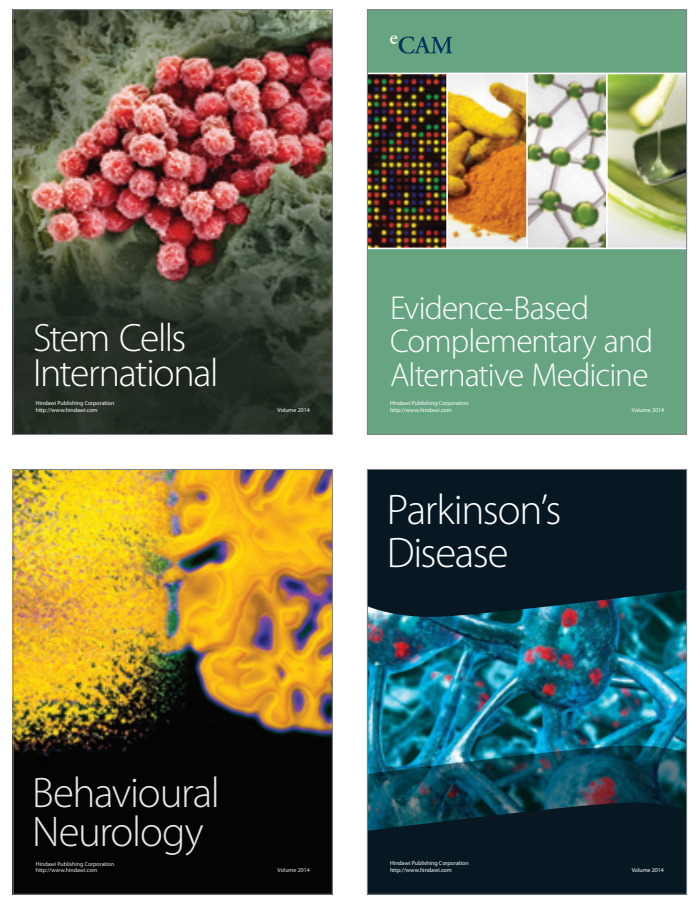
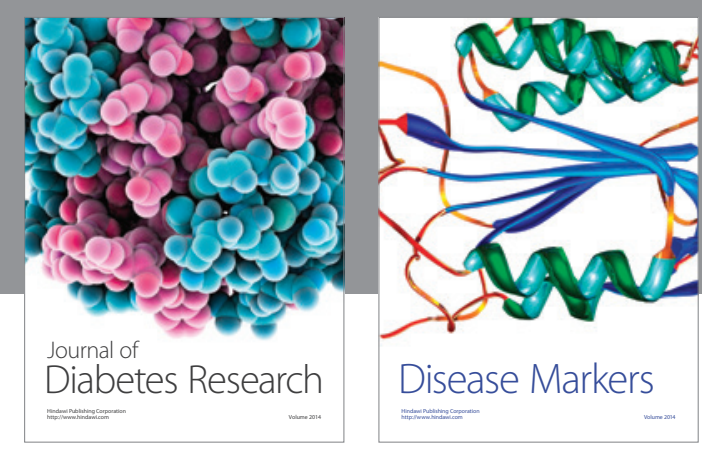

Disease Markers
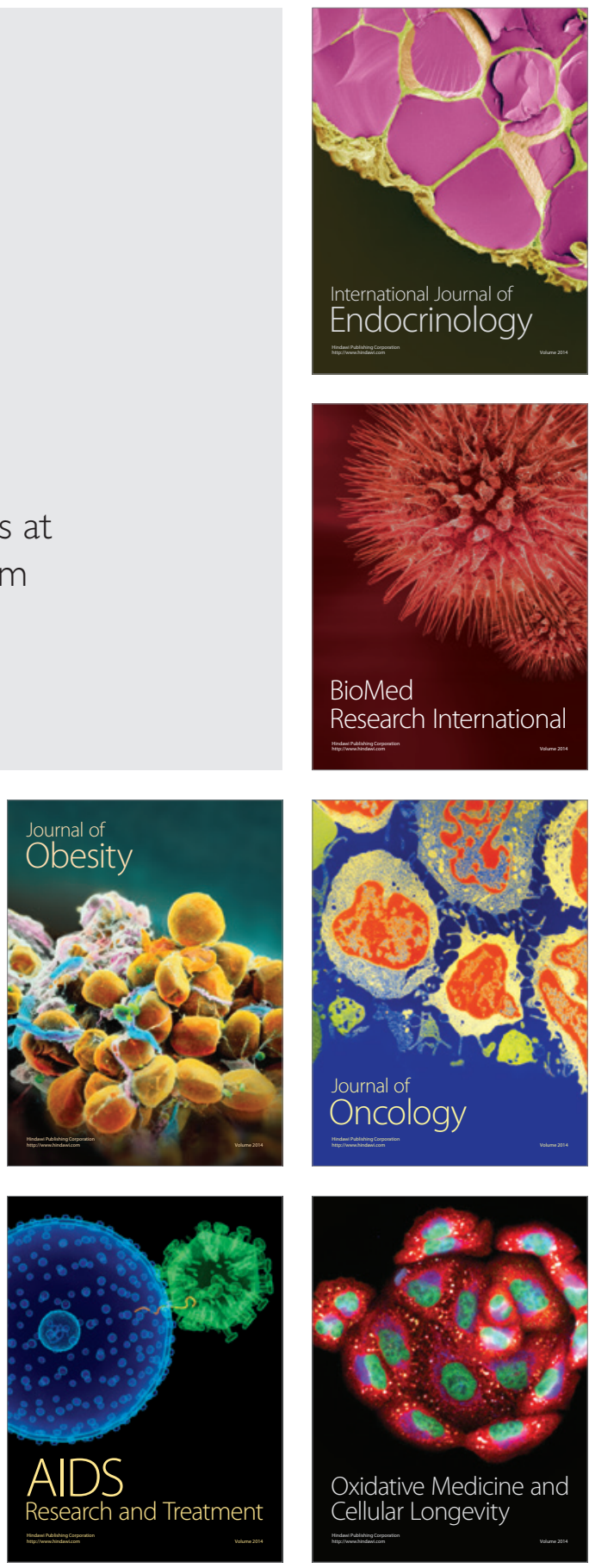